\title{
Risk Factors Associated with Cisplatin-Induced Nephrotoxicity in Patients with Advanced Lung Cancer
}

\author{
Takanori Miyoshi, ${ }^{* a}$ Nobuhiro Misumi, ${ }^{b}$ Mikako Hiraike, ${ }^{c}$ Yuki Mihara, ${ }^{d}$ Takashi Nishino, ${ }^{c}$ \\ Minako Tsuruta, ${ }^{a}$ Yosei Kawamata, ${ }^{a}$ Yoichi Hiraki, ${ }^{a}$ Aki Kozono, ${ }^{e}$ and Masao Ichikif \\ ${ }^{a}$ Department of Pharmacy, National Hospital Organization Beppu Medical Center; 1473 Uchikamado, Beppu, \\ Oita 874-0011, Japan: ${ }^{b}$ Department of Pharmacy, National Hospital Organization Kyushu Cancer Center; 3-1-1 \\ Notame, Minami-ku, Fukuoka 811-1395, Japan: ${ }^{c}$ Department of Pharmacy, National Hospital Organization Kyushu \\ Medical Center; 1-8-1 Jigyohama, Chuo-ku, Fukuoka 810-8563, Japan: ${ }^{d}$ Department of Pharmacy, National \\ Hospital Organization Miyakonojo Medical Center; 5033-1 Iwayoshi-cho, Miyakonojo, Miyazaki 885-0014, Japan: \\ ${ }^{e}$ Department of Pharmacy, National Hospital Organization Kumamoto Saisyunsou Hospital; 2659 Suya, Koshi, \\ Kumamoto 861-1196, Japan: and ${ }^{f}$ Department of Respirology, National Hospital Organization Kyushu Medical \\ Center; 1-8-1 Jigyohama, Chuo-ku, Fukuoka 810-8563, Japan. \\ Received June 13, 2016; accepted September 27, 2016
}

Cisplatin (CDDP) combination chemotherapy is widely administered to patients with advanced lung cancer. The dose depends on multiple factors, including whether the tumor is non-small-cell lung cancer (NSCLC) or small-cell lung cancer (SCLC). Although efficacy is limited by cisplatin-induced nephrotoxicity (CIN), little is known about the risk factors for this complication. The aim of this study was to identify the risk factors for CIN in patients with advanced lung cancer, both NSCLC and SCLC. We retrospectively reviewed clinical data for 148 patients who underwent initial chemotherapy including CDDP $\geq 50 \mathrm{mg} / \mathrm{m}^{2} \mathrm{per}$ patient per day for the first course at Kyushu Medical Center between October 2010 and September 2013. All data were collected from the electronic medical record system. Nephrotoxicity was defined as an increase in serum creatinine concentration of at least grade 2 during the first course of CDDP chemotherapy, as described by the National Cancer Institute Common Terminology Criteria for Adverse Events version 4.0. CIN was observed in nine patients. Univariate analysis revealed that cardiac disease and lower baseline serum albumin (Alb) values conferred a higher risk of nephrotoxicity $(\boldsymbol{p}<0.05)$. The cut-off value of Alb was $3.8 \mathrm{~g} /$ dL, calculated by receiver operating characteristics (ROC) curves. Multivariable logistic regression analysis revealed that cardiac disease (odds ratio $=11.7 ; p=0.002$ ) and hypoalbuminemia (odds ratio $=6.99 p=0.025$ significantly correlated with nephrotoxicity. In conclusion, cardiac disease and low baseline Alb values are possible risk factors for CIN.

Key words lung cancer; serum albumin; cardiac disease; cisplatin; risk factor; nephrotoxicity

Lung cancer is a leading cause of cancer death in Japan and other developed countries. ${ }^{1)}$ Platinum-based cytotoxic doublet chemotherapy is the main treatment for advanced lung cancer. Cisplatin (CDDP), an inorganic platinum chemotherapeutic drug, is widely administered either alone or in combination with other agents for the clinical treatment of various solid tumors, including lung, gastric, esophageal, bladder, and head and neck cancers. ${ }^{2}$ CDDP-based combination chemotherapy regimens have been used for first-line treatment of advanced lung cancer. ${ }^{3)}$ However, CDDP chemotherapy is often limited by its cumulative nephrotoxicity, ${ }^{4,5)}$ which is dose-dependent. ${ }^{6,7)}$ Standard procedures to reduce this risk include aggressive hydration with saline and simultaneous administration of diuretics such as furosemide and mannitol. ${ }^{8)}$ Despite these approaches, cisplatin-induced nephrotoxicity (CIN) still occurs. ${ }^{9-11)}$ The ability to identify patients at risk for nephrotoxicity would improve the efficacy and safety of CDDP. Although the risk factors for CIN have been extensively investigated and many factors have been reported, ${ }^{12-15}$ ) CIN prevention methods are not $100 \%$ successful. Therefore, assessment of renal function in patients treated with CDDP in the early course of treatment is necessary to prevent permanent damage to the kidneys.

The aims of this study were to investigate risk factors in patients undergoing early cycles of chemotherapy for ad- vanced lung cancer and to prevent permanent and continuous damage to the kidneys.

\section{PATIENTS AND METHODS}

Patients and Study Setting Patients were eligible for the study if they were diagnosed with advanced or recurrent lung cancer (non-small cell or small-cell) and scheduled to receive CDDP at a dose of $\geq 50 \mathrm{mg} / \mathrm{m}^{2}$ per patient per day at Kyushu Medical Center between October 2010 and September 2013. Patients who had a history of prior treatment with CDDP or whose renal function could not be evaluated, such as those with multiple organ failure, were excluded because of their unstable clinical status.

Data Collection and Assessment All data were collected from the hospital electronic medical record system (EGMAIN-GX version $5^{\circledR}$, Fujitsu, Tokyo, Japan). We collected information regarding patient gender, age, body mass index (BMI), histology, prior chemotherapy, Eastern Cooperative Oncology Group (ECOG) performance status (PS), clinical stage, complications, antineoplastic agents received, concurrent radiation, single dose per body-surface area of CDDP, hydration volume, use of analgesics, history of contrast studies, and baseline laboratory data. Patients with a history of angina, myocardial infarction, atrial fibrillation, arrhyth- 
mia, or valvular disease were defined as patients with cardiac disease in this study.

The laboratory data were from samples drawn on the day before chemotherapy. For evaluation of nephrotoxicity, the increase in serum creatinine ( $\mathrm{SCr}$ ) concentration was calculated as the maximum value within two weeks after CDDP administration minus the baseline value. For most patients, $\mathrm{SCr}$ was measured at least once every two weeks. The laboratory data included the levels of serum albumin (Alb), aspartate aminotransferase, alanine aminotransferase, $\gamma$-glutamyl transpeptidase, sodium, potassium, as well as SCr, creatinine clearance (CCr), hemoglobin, platelet count, and white blood cell count with differential. SCr was determined by an enzymatic method. $\mathrm{CCr}$ for males was calculated using the Cockcroft-Gault (CG) equation ${ }^{16)}$ :

$$
\begin{aligned}
\mathrm{CCr} & (\mathrm{mL} / \mathrm{min})^{\text {male }} \\
& =([140-\text { age }] \times \text { body weight }[\mathrm{kg}] / 72 \times \mathrm{SCr}(\mathrm{mg} / \mathrm{dL})
\end{aligned}
$$

$\mathrm{CCr}$ for women was then calculated using the following equation:

$$
\mathrm{CCr}(\mathrm{mL} / \mathrm{min})^{\text {female }}=\mathrm{CCr}(\mathrm{mL} / \mathrm{min})^{\text {male }} \times 0.85
$$

Definition of Nephrotoxicity In accordance with a previous study, ${ }^{17)}$ we adopted an increase in $\mathrm{SCr}$ as a measure of nephrotoxicity. The rationale for considering screening for early-stage nephrotoxicity includes detection of mild renal dysfunction due to aging. We excluded patients with renal dysfunction $\geq$ grade 1 before the first cycle of chemotherapy. Nephrotoxicity was defined according to the National Cancer Institute Common Terminology Criteria for Adverse Events (NCI CTCAE, version 4.0), after one chemotherapy cycle. ${ }^{18)}$ Because grade 1 nephrotoxicity is mild and often temporary, and chemotherapy is discontinued with grade 2 or higher nephrotoxicity in most cases, identification of risk factors for $\geq$ grade 2 is clinically important. Therefore, when the postCDDP level of $\mathrm{SCr}$ was $\geq 2$ grades higher than that before CDDP administration, the patient was placed in the nephrotoxicity group. The remaining patients were assigned to the non-nephrotoxicity group.

Statistical Analysis Differences in continuous data were compared using the Mann-Whitney $U$-test and differences in categorical data were compared using Fisher's exact test. Multiple logistic regression analysis of data from hematology and biochemical evaluations was used to analyze factors associated with nephrotoxicity. To identify factors associated with CIN, univariate analysis was performed using age, BMI, ECOG PS, clinical stage, hypertension, diabetes, cardiac disease (defined as the presence of angina, myocardial infarction, atrial fibrillation, arrhythmia, or valvular disease), concurrent radiation, CDDP dose, hydration volume (total amount of solution administered to prevent $\mathrm{CIN}$ ), Alb (normal range in our hospital is $3.8-5.3 \mathrm{~g} / \mathrm{dL}$ ), sodium, potassium $(3.5-4.8 \mathrm{mEq} / \mathrm{L})$, and $\mathrm{CCr}$ (male: $90-120 \mathrm{~mL} / \mathrm{min}$, female: $80-110 \mathrm{~mL} / \mathrm{min}$ ) as independent variables. Differences between each factor were tested for statistical significance $(p<0.15)$ using Fisher's exact test. Variables with a $p<0.15$ in the initial analysis were included, and only those variables with a $p<0.05$ remained in the final model in the multivariate logistic regression analysis. The magnitude of the association was expressed as an odds ratio (OR) and 95\% confidence interval (95\% CI). The sensitivity, specificity, and cut-off values for factors obtained by univariate analysis were estimated by calculating the area under the receiver-operator characteristics (ROC) curve. The area under the curve $(A U C)$ was calculated to determine the inherent ability of the test to discriminate between nephrotoxicity and non-nephrotoxicity. All statistical analyses were performed with commercial software $\left(\mathrm{EZR}^{\circledR}\right.$, Saitama Medical Center, Jichi Medical University, http://www.jichi.ac.jp/saitama-sct/SaitamaHP.files/statmedEN.html), which is a graphical user interface for R (The R Foundation for Statistical Computing, version 2.13.0). More precisely, it is a modified version of $\mathrm{R}$ commander (version 1.8-4) ${ }^{19)}$ designed to add statistical functions frequently used in biostatistics. A $p<0.05$ was considered statistically significant.

Ethics This study was approved by the ethics committee of the National Hospital Organization Kyushu Medical Center. The study was conducted in accordance with the ethical guidelines of the Declaration of Helsinki and the guidelines of the International Conference on Harmonization Good Clinical Practice. $^{20,21)}$

\section{RESULTS}

Patient Characteristics A total of 148 patients who had undergone therapy with CDDP were included in the study. Baseline characteristics and biochemical parameters of these 148 patients are summarized in Tables 1 and 2. CIN was observed in nine $(6.1 \%)$ patients, with six $(4.1 \%)$ and three $(2.0 \%)$ cases of grade 2 and 3, respectively. All patients who developed CIN were male. The median (IQR: interquartile range) of CDDP dosage and CCr was $74.5 \mathrm{mg} / \mathrm{m}^{2}\left(68.2-75.0 \mathrm{mg} / \mathrm{m}^{2}\right)$ and $86.8 \mathrm{~mL} / \mathrm{min}(75.1-102.6 \mathrm{~mL} / \mathrm{min})$, respectively. Compared with patients without CIN, the proportion of patients who had cardiac disease was significantly higher in patients with CIN (44.4\% vs. 7.2\%, $p=0.005$ ). In addition, median baseline Alb values were significantly different between the nephrotoxicity and non-nephrotoxicity groups $(3.4 \mathrm{~g} / \mathrm{dL} v s .3 .9 \mathrm{~g} / \mathrm{dL}$, respectively, $p=0.007$ ).

CCr before and after Administration of CDDP Table 3 shows $\mathrm{CCr}$ before and after administration of CDDP. The mean $\mathrm{CCr}$ of the non-nephrotoxic group of patients was $86.9 \mathrm{~mL} / \mathrm{min}$ before administration and $73.4 \mathrm{~mL} / \mathrm{min}$ after administration $(p=0.446)$. Values in the nephrotoxic group of patients were 84.3 and $22.8 \mathrm{~mL} / \mathrm{min}$, respectively $(p<0.001)$.

Univariate Analysis Univariate analysis revealed that cardiac disease (OR: 10.3, 95\% CI: 2.39-44.6, $p=0.005$ ), baseline Alb values (OR: 6.2, 95\% CI: $1.25-31.1 ; p=0.03)$ and $\mathrm{K}$ value (OR: $4.85,95 \% \mathrm{CI}$ : $1.08-21.7, p=0.06$ ) were significantly associated with CIN (Table 4).

Multivariate Analysis We carried out a multivariate logistic regression analysis using the factors of cardiac disease and Alb values which remained by univariate analysis. The results of the multivariate logistic regression analysis are shown in Table 5. Multivariate logistic regression analysis suggested that patients who had cardiac disease (OR: 11.70, 95\% CI: 2.14-57.2, $p=0.002$ ) and Alb values (OR: 6.99, 95\% CI: $1.28-38.2, p=0.025)$ had a higher risk of CIN.

ROC Analysis To estimate the predictive value of $\mathrm{Alb}$ level for CIN, the ROC curve and $A U C$ obtained by univariate analysis of Alb was calculated. The cut-off value of Alb calculated by the ROC curve was $3.8 \mathrm{~g} / \mathrm{dL}$ (Fig. 1). The $A U C$ was 0.77 , which was evaluated as having moderate accuracy for 
Table 1. Patient Characteristics

\begin{tabular}{|c|c|c|c|c|}
\hline \multirow[b]{2}{*}{ Parameter } & & \multicolumn{2}{|c|}{ Nephrotoxicity } & \multirow[b]{2}{*}{$p$ Value } \\
\hline & & $\begin{array}{c}- \\
n=139\end{array}$ & $\begin{array}{c}+ \\
n=9\end{array}$ & \\
\hline Gender & Male/Female & $94 / 45$ & $9 / 0$ & $0.058^{a)}$ \\
\hline Age (years) & Median (IQR) & $64(59.5-69)$ & $61(60-71)$ & $0.751^{b)}$ \\
\hline BMI $\left(\mathrm{kg} / \mathrm{m}^{2}\right)$ & Median (IQR) & $22.2(20.2-24.2)$ & $20.6(18.0-25.1)$ & $0.591^{b)}$ \\
\hline \multirow[t]{3}{*}{ Histology } & Small cell & $33(23.7 \%)$ & $2(22.2 \%)$ & $1.000^{a)}$ \\
\hline & Non-small cell & $96(69.1 \%)$ & $5(55.6 \%)$ & $0.466^{a)}$ \\
\hline & Others & $10(7.2 \%)$ & $2(22.2 \%)$ & $0.158^{a)}$ \\
\hline \multirow[t]{2}{*}{ ECOG PS } & 0 & $93(66.9 \%)$ & $5(55.6 \%)$ & $0.487^{a)}$ \\
\hline & 1,2 & $46(33.1 \%)$ & $4(44.4 \%)$ & \\
\hline \multirow[t]{2}{*}{ Clinical stage } & III & $42(30.2 \%)$ & $3(33.3 \%)$ & $1.000^{a)}$ \\
\hline & IV, Recurrence & $97(69.8 \%)$ & $6(66.7 \%)$ & \\
\hline \multirow[t]{2}{*}{ Prior chemotherapy } & 0 & $121(87.1 \%)$ & $9(100 \%)$ & $0.601^{a)}$ \\
\hline & $\geq 1$ & $18(12.9 \%)$ & $0(0 \%)$ & \\
\hline \multirow[t]{4}{*}{ Comorbidities } & Hypertension & $50(36.0 \%)$ & $5(55.6 \%)$ & $0.293^{a)}$ \\
\hline & Diabetes & $16(11.5 \%)$ & $2(22.2 \%)$ & $0.300^{a)}$ \\
\hline & Hyperlipidemia & $27(19.4 \%)$ & $0(0 \%)$ & $0.366^{a)}$ \\
\hline & Cardiac disease & $10(7.2 \%)$ & $4(44.4 \%)$ & $0.005^{a)}$ \\
\hline \multirow[t]{7}{*}{ Co-administered drugs } & Pemetexed \pm Bevacizumab & $78(56.1 \%)$ & $4(44.4 \%)$ & $0.513^{a)}$ \\
\hline & Docetaxel & $11(7.9 \%)$ & $1(11.1 \%)$ & $0.543^{a)}$ \\
\hline & Vinorelbine & $13(9.4 \%)$ & $1(11.1 \%)$ & $1.000^{a)}$ \\
\hline & Irinotecan & $20(14.4 \%)$ & $1(11.1 \%)$ & $1.000^{a)}$ \\
\hline & Etoposide & $14(10.0 \%)$ & $1(11.1 \%)$ & $1.000^{a)}$ \\
\hline & Gemcitabine & $2(1.4 \%)$ & $1(11.1 \%)$ & $0.173^{a)}$ \\
\hline & Tegafur $\cdot$ gimestat $\cdot$ otastat & $1(0.7 \%)$ & $0(0 \%)$ & $1.000^{a)}$ \\
\hline \multirow[t]{2}{*}{ Concurrent radiation } & + & $26(18.7 \%)$ & $2(22.2 \%)$ & $0.679^{a)}$ \\
\hline & - & $113(81.3 \%)$ & $7(77.8 \%)$ & \\
\hline Cisplatin dose $\left(\mathrm{mg} / \mathrm{m}^{2}\right)$ & Median (IQR) & $74.5(68.2-75.0)$ & $75(73.2-79.1)$ & $0.290^{b)}$ \\
\hline Hydration volume $(\mathrm{mL})$ & Median (IQR) & $5000(5000-6000)$ & $5000(5000-5000)$ & $0.273^{b)}$ \\
\hline Use of analgesics (including NSAIDs) & & $40(28.8 \%)$ & $3(33.3 \%)$ & $0.720^{a)}$ \\
\hline History of contrast studies & & $139(100 \%)$ & $9(100 \%)$ & - \\
\hline
\end{tabular}

a) Fisher's exact test. b) Mann-Whitney U-test. IQR; interquartile range, BMI; body mass index, ECOG; Eastern Cooperative Oncology Group, PS; performance status; Hydration volume; total amount of solution administered during cisplatin infusion, NSAIDs; Non-steroidal anti-inflammatory drugs. $*$ Significance level was set at $p<0.05$.

Table 2. Patient Baseline Biochemical Parameters

\begin{tabular}{lccc}
\hline \hline \multirow{2}{*}{ Parameter } & \multicolumn{2}{c}{ Nephrotoxicity } & \multirow{2}{*}{$p$ Value } \\
\cline { 2 - 3 } & \multicolumn{1}{c}{-} & + & \\
\hline Alb $(\mathrm{g} / \mathrm{dL})$ & $3.9(3.5-4.2)$ & $3.4(3.2-3.7)$ & $0.007^{*}$ \\
$\mathrm{AST}(\mathrm{IU} / \mathrm{L})$ & $22(18-28)$ & $22(22-32)$ & 0.193 \\
$\mathrm{ALT}(\mathrm{IU} / \mathrm{L})$ & $21(15-28)$ & $25(13-32)$ & 0.773 \\
$\gamma$-GTP $(\mathrm{IU} / \mathrm{L})$ & $33(20-53)$ & $29(21-75)$ & 0.610 \\
$\mathrm{Na}(\mathrm{mEq} / \mathrm{L})$ & $139(138-140)$ & $138(137-141)$ & 0.737 \\
$\mathrm{~K}(\mathrm{mEq} / \mathrm{L})$ & $4.2(4.0-4.5)$ & $4.2(4.1-4.3)$ & 0.667 \\
$\mathrm{CCr}(\mathrm{mL} / \mathrm{min})^{*}$ & $86.9(75.5-102.7)$ & $84.3(73.1-92.4)$ & 0.446 \\
$\mathrm{WBC}\left(\times 10^{3} / \mu \mathrm{L}\right)$ & $6.9(5.7-8.1)$ & $8.1(5.1-9.8)$ & 0.697 \\
$\mathrm{Hb}(\mathrm{g} / \mathrm{dL})$ & $12.9(12.1-13.9)$ & $12.7(12.4-13.0)$ & 0.340 \\
$\mathrm{PLT}\left(\times 10^{3} / \mu \mathrm{L}\right)$ & $280(232-345)$ & $300(240-465)$ & 0.537 \\
$\mathrm{Neut}\left(\times 10^{3} / \mu \mathrm{L}\right)$ & $4.27(3.58-5.58)$ & $5.56(2.71-6.90)$ & 0.785 \\
$\mathrm{Ly}\left(\times 10^{2} / \mu \mathrm{L}\right)$ & $1.45(1.12-1.92)$ & $1.58(0.73-1.63)$ & 0.404 \\
$\mathrm{Mono}\left(\times 10^{2} / \mu \mathrm{L}\right)$ & $4.40(3.52-5.38)$ & $4.65(3.52-6.67)$ & 0.739 \\
\hline
\end{tabular}

All quantities are expressed as median (IQR), *Calculated by the Cockcroft-Gault equation, Alb, albumin; AST, aspartate aminotransferase; ALT, alanine aminotransferase; $\gamma$-GTP, $\gamma$-glutamyl transpeptidase; CCr, creatinine clearance rat. *Significance level was set at $p<0.05$.

detecting some events. Using this cut-off value, the sensitivity and specificity of Alb level to detect the risk for CIN were 56.8 and $88.9 \%$, respectively.
Table 3. Creatinine Clearance $(\mathrm{CCr})$ before and after Administration of CDDP

\begin{tabular}{|c|c|c|c|}
\hline \multirow[t]{2}{*}{ Parameter } & $\begin{array}{c}\text { Before } \\
\text { administration }\end{array}$ & $\begin{array}{c}\text { After } \\
\text { administration }\end{array}$ & \multirow{2}{*}{$\begin{array}{c}p \\
\text { Value }\end{array}$} \\
\hline & Median (IQR) & Median (IQR) & \\
\hline Non nephrotoxicity patients & $86.9(75.5-102.8)$ & $73.4(60.2-86.0)$ & 0.446 \\
\hline Nephrotoxicity patients & $84.3(73.9-92.4)$ & $22.8(14.1-32.9)$ & $0.001 *$ \\
\hline
\end{tabular}

\section{DISCUSSION}

Although CDDP dosing regimens for individual patients have been established using recommended parameters ${ }^{22)}$ such as dose per body surface area and the results of hematologic and biochemical examinations, ${ }^{23)}$ the main factors linked to renal failure in lung cancer patients treated with CDDP remain unclear. The main risk factors for CIN such as older age, female gender, smoking, hypoalbuminemia, diabetes mellitus, and cardiovascular disease have been extensively studied..$^{10,17,24)}$ However, these risk factors are not considered when making clinical decisions about treatment continua- 
Table 4. Univariate Analysis for Cisplatin-Induced Nephrotoxicity

\begin{tabular}{lclc}
\hline \hline Factors & Odds ratio & $95 \%$ CI & $p$ Value \\
\hline Age $($ years $)$ & 0.43 & $0.10-1.77$ & 0.430 \\
BMI $\left(\mathrm{kg} / \mathrm{m}^{2}\right)$ & 1.23 & $0.32-4.78$ & 1.000 \\
ECOG PS, $\geq 1$ & 1.62 & $0.41-0.31$ & 0.488 \\
Clinical stage, III & 0.87 & $0.21-3.63$ & 0.870 \\
Hypertension & 2.23 & $0.57-8.67$ & 0.293 \\
Diabetes & 2.20 & $0.42-11.50$ & 0.300 \\
Cardiac disease & 10.32 & $2.39-44.61$ & $0.005^{*}$ \\
Concurrent radiation & 1.24 & $0.24-6.33$ & 0.679 \\
Cisplatin dose $\left(\mathrm{mg} / \mathrm{m}^{2}\right)$ & 1.92 & $0.46-7.97$ & 0.497 \\
Hydration volume $(\mathrm{mL})$ & 0.30 & $0.03-2.87$ & 0.318 \\
Alb $(\mathrm{g} / \mathrm{dL})$ & 6.23 & $1.25-31.14$ & $0.028^{*}$ \\
Na $(\mathrm{mEq} / \mathrm{L})$ & 1.54 & $0.37-6.51$ & 0.691 \\
$\mathrm{~K}(\mathrm{mEq} / \mathrm{L})$ & 4.85 & $1.08-21.70$ & $0.059 *$ \\
CCr $(\mathrm{mL} / \mathrm{min})$ & 1.43 & $0.34-5.96$ & 0.737 \\
\hline
\end{tabular}

CI, confidence interval; BMI, body mass index; ECOG, Eastern Cooperative Oncology Group; PS, performance status; Alb, albumin; $\mathrm{CCr}$, creatinine clearance rate; Hydration volume, total amount of solution administered during cisplatin infusion $*$ Significantly different, $p<0.05$. $p$-values were calculated from the Fisher's exact test for categorical.

Table 5. Multivariate Analysis for CDDP Nephrotoxicity

\begin{tabular}{lrrr}
\hline \hline Factor & Odds ratio & $95 \% \mathrm{CI}$ & $p$ Value \\
\hline Alb & 6.99 & $1.28-38.2$ & $0.025^{*}$ \\
Cardiac disease & 11.70 & $2.14-57.2$ & $0.002 *$ \\
\hline
\end{tabular}

$95 \%$ CI, 95\% confidence interval; Alb, albumin. * Significance level was set at $p<0.05$.

tion, because they are non-numerical objective phenomena and cannot be used for treatment planning. Only conventional criteria such as body surface area (BSA) or CCr level are used for administration plans. ${ }^{25)}$ In this study, heart disorders and hypoalbuminemia were risk factors for CIN, similar to recent findings. ${ }^{24)}$ Thus we clarified the threshold of the Alb value quantitatively. Multivariate logistic regression analysis suggested that cardiac disease and baseline Alb were significant risk factors. In this study, we suggested that low Alb value is a risk factor of renal dysfunction, and several previous reports support these results. ${ }^{12,26)}$ Although sensitivity was definitely not high (56.8\%) when using Alb values, a high Alb value is less likely to be a risk of renal dysfunction. Thus, close monitoring of changes in Alb value during CDDP treatment may prevent the development of CIN in these patients.

CIN remains a clinical problem in $28-42 \%$ of patients. ${ }^{27)}$ Most cases are transient and reversible, but irreversible CIN sometimes occurs and can limit treatment. Generally, in patients with cardiac disease, a reduction in renal perfusion caused by decreased cardiac output may affect the clearance of drugs eliminated by renal clearance, such as CDDP, ABK (arbekacin) and TEIC (teicoplanin). Ishida et al. ${ }^{28)}$ reported that the clearance of $\mathrm{ABK}$, an antibiotic that is eliminated by renal clearance, was significantly decreased in patients with cardiac disease compared with those without cardiac disease. Renal excretion of TEIC and vancomycin is also decreased in patients with heart disorders. ${ }^{29,30)}$ Thus, decreased renal perfusion associated with cardiac disease may influence the clearance of CDDP. A decrease in renal blood flow due to heart disorders reduces renal clearance, inducing renal dysfunction. Although the patients with cardiac disease developed renal

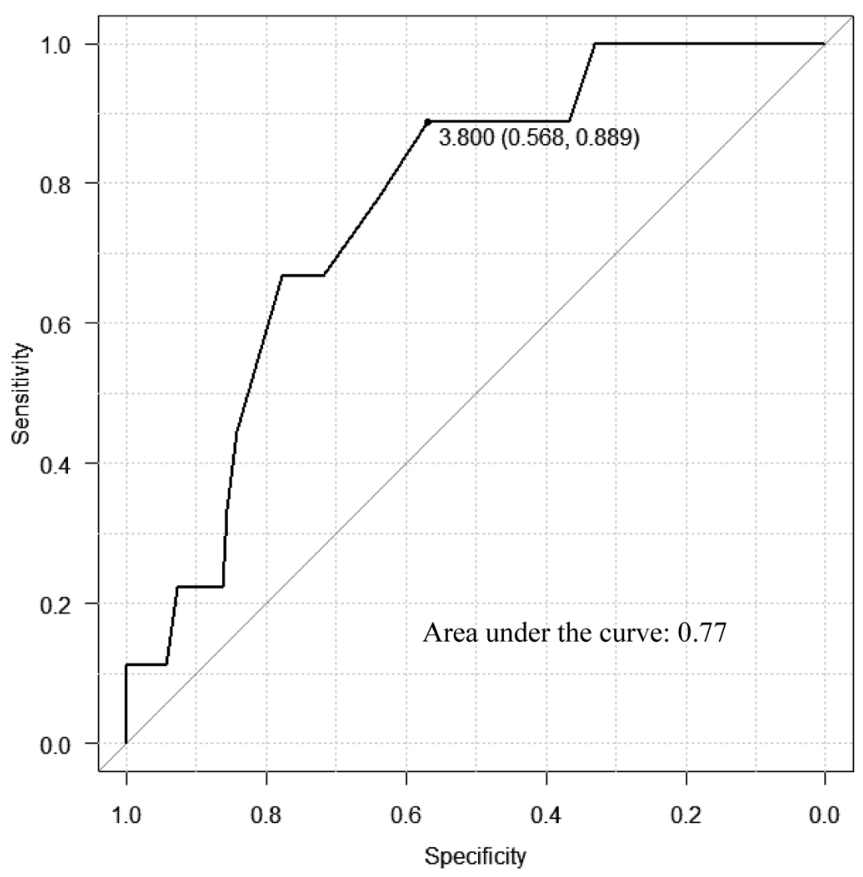

Fig. 1. Receiver Operating Characteristic (ROC) Analysis

The sensitivity, specificity, and cut-off values using ROC curves between Alb level (independent variable, $x$ ) and the probability of renal failure development (dependent variable, $y$ ).

dysfunction (Table 1, $p=0.005$ ), baseline $\mathrm{CCr}$ did not differ between patients with and without cardiac disease (Table 2, $p=0.446)$. In this study, the number of patients with cardiac disease was extremely small. Most of the subjects were elderly, with the reduction in muscle mass that accompanies aging, and 25 patients had BMI $\leq 18.5$. The muscle mass greatly affects serum creatinine, resulting in glomerular filtration rate (GFR) being overestimated, because the CCr level calculated by the CG equation depends on SCr. Therefore, we think that mild renal insufficiency in patients with low muscle mass might be missed, because GFR is overestimated, CIN is considered to primarily affect the proximal tubule cells. ${ }^{31)}$ On this basis, prevention is based on the use of hydration combined with diuretics to reduce CDDP contact time with the proximal tubule. Mathe et al. ${ }^{32)}$ also reported that CIN was aggravated by comorbidity with cardiac disease in lung cancer patients.

The second independent risk factor we identified for CIN was low baseline Alb values. CIN is considered to be caused by lower levels of plasma proteins binding CDDP. One study reported that approximately $98 \%$ of CDDP immediately binds to plasma proteins, mainly $\mathrm{Alb},{ }^{33)}$ while various other studies demonstrated that CIN is related to the peak plasma CDDP concentration and/or the area under the plasma CDDP concentration-time curve of unbound CDDP. ${ }^{34-36}$ ) These findings suggest that nephrotoxicity may result from decreased protein binding due to low serum Alb.

In patients with renal dysfunction, the pharmacokinetics of CDDP vary with the degree of renal dysfunction. Adjustment of CDDP dose is made according to $\mathrm{CCr}$ level. ${ }^{27)} \mathrm{We}$ hypothesize that decreased binding of CDDP in the presence of low Alb values can lead to renal dysfunction, particularly in patients with compromised renal perfusion due to cardiac dysfunction. By analyzing the association between Alb level (independent variable, $x$ ) and the probability of renal failure 
development (dependent variable, y) using ROC curves, we determined that the cut-off value for the development of renal dysfunction was an Alb value of $3.8 \mathrm{~g} / \mathrm{dL}$. As seven of the nine patients with $\mathrm{CIN}$ had Alb values $<3.8 \mathrm{~g} / \mathrm{dL}$, our preliminary results suggest that a lower baseline Alb value is a risk factor for CIN.

Previous studies have reported ${ }^{12,26)}$ on the relationship of CIN and Alb value. It has been suggested ${ }^{12,37)}$ that hypoalbuminemia reduces platinum excretion due to its effect on peritubular oncotic pressure and on the half-life of the CDDP, because the protein binding rate of the CDDP decreases and free CDDP increases, resulting in greater exposure to free CDDP. Furthermore, Mizuno et al. reported ${ }^{36)}$ that lower blood pressure was significantly correlated with hypoalbuminemia, and reported hypoalbuminemia and hypotension as nephropathic risk factors. These reports support our finding that a decrease in blood pressure according to abnormalities of cardiac output and ejection fraction, and an increase in free CDDP with hypoalbuminemia are risk factors for nephrotoxicity in CDDP treatment. Especially regarding free CDDP concentration, in order to attain more effective CDDP chemotherapy with minimum nephrotoxicity, the present pharmacokinetic and pharmacodynamic studies suggest that the $C_{\max }$ or steady-state plasma level of unchanged free CDDP should be maintained between 1.5 and $2 \mu \mathrm{g} / \mathrm{mL}^{35)}$ Although our findings are preliminary, our ROC analysis suggests that an Alb level $<3.8 \mathrm{~g} /$ $\mathrm{dL}$ may lead to the development of nephrotoxicity. The findings in this study are similar to those of previous studies, ${ }^{12-15}$ ) therefore confirming that decreased renal perfusion and hypoalbuminemia are nephropathic risk factors associated with CDDP in lung cancer chemotherapy. However, our study was unable to elucidate criteria for dose adjustment according to cardiac disease and Alb values. Further investigation of this is necessary, as is the establishment of more effective strategies to prevent CIN for patients with these risk factors.

Several limitations of the study warrant mention. First, only a small number of patients with lung cancer with chemotherapy using CDDP were enrolled into this study. Although the small study size dictates that our results should be considered preliminary, the trends identified here support the need for a larger study. Second, several previous studies reported that intravenous supplementation with $\mathrm{Mg}$ (magnesium) can prevent CIN. ${ }^{26,38,39)}$ Because our treatment protocol did not include $\mathrm{Mg}$ administration, we cannot state its efficacy in patients with these risk factors. Third, we defined the patients with a history of the cardiac disease such as angina and myocardial infarction as patients with cardiac disease in this study. However, quantifiable data (e.g., cardiac output and an ejection fraction) were not available. Therefore we defined heart disorders by medical history. Fourth, because our study was conducted under a retrospective design, we were unable to fully evaluate the incidence and intensity of nonhematologic toxicities. Nonhematologic toxicities, such as nausea, vomiting, and diarrhea might be correlated with an increased risk for CIN. Finally, although the use of a drug which induces renal failure may be limited by the discretion of the physician, most patients with lung cancer routinely take non-steroidal anti-inflammatory drugs (NSAIDs) and narcotic analgesics for control of pain, and may undergo CT scans using contrast media to evaluate the efficacy of the chemotherapy. Although NSAIDs pose little threat of renal insult in normal, healthy persons at therapeutic dosages, NSAIDs administration to susceptible persons may cause decrements in renal plasma flow and glomerular filtration rate within hours. ${ }^{40)}$ Sendur et al. suggested that radiographic procedures with intravenous contrast material should be delayed for at least 1 week in patients receiving chemotherapy with CDDP to prevent nephrotoxicity. ${ }^{41)}$ In the current study, all patients underwent CT scans using contrast media at an interval of about 2 weeks to 1 month after chemotherapy, which was thought to be a sufficient period to eliminate effects of the contrast media. The rates of use of analgesics were 28.8 and $33.3 \%$ in the nonnephrotoxicity and nephrotoxicity groups, respectively (Table 1). These agents were long-term analgesics taken from before chemotherapy initiation, and the dose was not changed during treatment. Therefore, comorbidities relevant to nephrotoxicity due to NSAIDs and contrast agents were not assessed in the study. Such drug-induced nephrotoxicity, including acute tubular necrosis, inflammation, and altered hemodynamics such as reduction of renal blood flow by the inhibition of prostaglandin, might be associated with an increased risk for CIN. ${ }^{42}$ ) In conclusion, our findings suggest that cardiac disease and baseline Alb values $<3.8 \mathrm{~g} / \mathrm{dL}$ are important risk factors for CIN in patients with advanced lung cancer. Thus, close monitoring of $\mathrm{SCr}$ values during CDDP treatment is recommended for patients with these risk factors.

Conflict of Interest The authors declare no conflict of interest.

\section{REFERENCES}

1) Siegel R, DeSantis C, Virgo K, Stein K, Mariotto A, Smith $T$, Cooper D, Gansler T, Lerro C, Fedewa S, Lin C, Leach C, Cannady RS, Cho H, Scoppa S, Hachey M, Kirch R, Jemal A, Ward E. Cancer treatment and survivorship statistics. CA Cancer J. Clin., 62, 220-241 (2012).

2) Rozencweig $\mathrm{M}$, von Hoff DD, Slavik M, Muggia FM. cisDiamminedichloroplatinum(II). A new anticancer drug. Ann. Intern. Med., 86, 803-812 (1977).

3) SW J, PJ OD. Pharmacology of cancer chemotherapy. Cisplatin and its analogues. (De Vita VT Jr, Hellman S, Rosenberg SA ed.) Cancer, Principles and Practice of Oncology, 7th ed. Philadelphia, Lippincott Williams \& Wilkins, Philadelphia (2005).

4) Wang D, Lippard SJ. Cellular processing of platinum anticancer drugs. Nat. Rev. Drug Discov., 4, 307-320 (2005).

5) Pabla N, Dong Z. Cisplatin nephrotoxicity: mechanisms and renoprotective strategies. Kidney Int., 73, 994-1007 (2008).

6) Lehane D, Winston A, Gray R, Daskal Y. The effect of diuretic pre-treatment on clinical, morphological and ultrastructural cisplatinum induced nephrotoxicity. Int. J. Radiat. Oncol. Biol. Phys., 5, 1393-1399 (1979).

7) Madias NE, Harrington JT. Platinum nephrotoxicity. Am. J. Med., 65, 307-314 (1978).

8) Ries F, Klastersky J. Nephrotoxicity induced by cancer chemotherapy with special emphasis on cisplatin toxicity. Am. J. Kidney Dis. 8, 368-379 (1986).

9) Kovach JS, Moertel CG, Schutt AJ, Reitemeier RG, Hahn RG. Phase II study of cis-diamminedichloroplatinum (NSC-119875) in advanced carcinoma of the large bowel. Cancer Chemother. Rep., 57, 357-359 (1973).

10) de Jongh FE, van Veen RN, Veltman SJ, de Wit R, van der Burg ME, van den Bent MJ, Planting AS, Graveland WJ, Stoter G, Verweij J. Weekly high-dose cisplatin is a feasible treatment option: 
analysis on prognostic factors for toxicity in 400 patients. $\mathrm{Br} . \mathrm{J}$. Cancer, 88, 1199-1206 (2003).

11) dos Santos NA, Carvalho Rodrigues MA, Martins NM, dos Santos AC. Cisplatin-induced nephrotoxicity and targets of nephroprotection: an update. Arch. Toxicol., 86, 1233-1250 (2012).

12) Nanji AA, Stewart DJ, Mikhael NZ. Hyperuricemia and hypoalbuminemia predispose to cisplatin-induced nephrotoxicity. Cancer Chemother. Pharmacol., 17, 274-276 (1986).

13) Miller RP, Tadagavadi RK, Ramesh G, Reeves WB. Mechanisms of cisplatin nephrotoxicity. Toxins (Basel), 2, 2490-2518 (2010).

14) Kreusser W, Herrmann R, Tschöpe W, Ritz E. Nephrological complications of cancer therapy. Contrib. Nephrol., 33, 223-238 (1982).

15) Fukasawa H, Furuya R, Yasuda $H$, Yamamoto $T$, Hishida A, Kitagawa M. Anti-cancer agent-induced nephrotoxicity. Anti-cancer Agents Med. Chem., 14, 921-927 (2014).

16) Haim N, Oman SD, Galai N, Burde B, Nathan S, Catane R. Estimation of creatinine clearance without 24-hour urine collection-a useful guide during cisplatin therapy. Acta Oncol., 32, 409-412 (1993).

17) Stewart DJ, Dulberg CS, Mikhael NZ, Redmond MD, Montpetit VA, Goel R. Association of cisplatin nephrotoxicity with patient characteristics and cisplatin administration methods. Cancer Chemother. Pharmacol., 40, 293-308 (1997).

18) DCTD N, NIH, DHHS. Common Terminology Criteria for Adverse Events, Version 4.03, DCTD, NCI, NIH, DHHS. 14 June 2010 (2010).

19) Kanda Y. Investigation of the freely available easy-to-use software 'EZR' for medical statistics. Bone Marrow Transplant., 48, 452-458 (2013).

20) World Medical Association Inc. Declaration of Helsinki. Ethical principles for medical research involving human subjects. J. Indian Med. Assoc., 107, 403-405 (2009).

21) Dixon JR Jr. The International Conference on Harmonization Good Clinical Practice guideline. Qual. Assur., 6, 65-74 (1998).

22) Gurney H. How to calculate the dose of chemotherapy. Br. J. Cancer, 86, 1297-1302 (2002).

23) Japanese Society of Nephrology. "Nephrotoxicity risk factor by CDDP in guidelines.”: 〈www.jsn.or.jp/guideline/pdf/2016-cancerguideline.pdf), cited 06 July, 2016.

24) Mizuno T, Ishikawa K, Sato W, Koike T, Kushida M, Miyagawa Y, Yamada K, Hirata S, Imai E, Noda Y. The risk factors of severe acute kidney injury induced by cisplatin. Oncology, 85, 364-369 (2013).

25) Kutluk Cenik B, Sun H, Gerber DE. Impact of renal function on treatment options and outcomes in advanced non-small cell lung cancer. Lung Cancer, 80, 326-332 (2013).

26) Mizuno T, Hayashi T, Shimabukuro $Y$, Murase M, Hayashi $H$, Ishikawa K, Takahashi K, Yuzawa Y, Yamada S, Nagamatsu T. Lower blood pressure-induced renal hypoperfusion promotes cisplatin-induced nephrotoxicity. Oncology, 90, 313-320 (2016).

27) Bodnar L, Wcislo G, Gasowska-Bodnar A, Synowiec A, SzarlejWcislo K, Szczylik C. Renal protection with magnesium subcarbonate and magnesium sulphate in patients with epithelial ovarian cancer after cisplatin and paclitaxel chemotherapy: a randomised phase II study. Eur. J. Cancer, 44, 2608-2614 (2008).

28) Ishida SYK, Umeda N, Maeda K, Ohshima T, Nakai Y. Arbekacin serum pharmacokinetics in patients with cardiac disease. Jpn. J. Chemother., 59, 374-378 (2011).
29) Fukumoto K, Shimamoto Y, Tsuchishita Y, Kusumoto M, Ueno K. Evaluation of teicoplanin pharmacokinetics in neonates and infanst. Jpn. J. Ther. Drug Monitor., 24, 128-132 (2007).

30) Teramachi $H$, Hatakeyama $H$, Matsushita R, Imai Y, Miyamoto K, Tsuji A. Evaluation of predictability for vancomycin dosage regimens by the Bayesian method with Japanese population pharmacokinetic parameters. Biol. Pharm. Bull., 25, 1333-1338 (2002).

31) Townsend DM, Deng M, Zhang L, Lapus MG, Hanigan MH. Metabolism of cisplatin to a nephrotoxin in proximal tubule cells. $J$. Am. Soc. Nephrol., 14, 1-10 (2003).

32) Máthé C, Bohács A, Duffek L, Lukácsovits J, Komlosi ZI, Szondy K, Horváth I, Müller V, Losonczy G. Cisplatin nephrotoxicity aggravated by cardiovascular disease and diabetes in lung cancer patients. Eur. Respir. J., 37, 888-894 (2011).

33) Horiuchi M, Inuyama $Y$, Kohno N, Mashino S, Fujii M. Pharmacokinetics of cis-dichlorodiammineplatinum(II). Gan To Kagaku Ryoho, 9, 632-637 (1982).

34) Reece PA, Stafford I, Russell J, Khan M, Gill PG. Creatinine clearance as a predictor of ultrafilterable platinum disposition in cancer patients treated with cisplatin: relationship between peak ultrafilterable platinum plasma levels and nephrotoxicity. J. Clin. Oncol., 5, 304-309 (1987).

35) Nagai N, Kinoshita M, Ogata H, Tsujino D, Wada Y, Someya K, Ohno T, Masuhara K, Tanaka Y, Kato K, Nagai H, Yokoyama A, Kurita Y. Relationship between pharmacokinetics of unchanged cisplatin and nephrotoxicity after intravenous infusions of cisplatin to cancer patients. Cancer Chemother. Pharmacol., 39, 131-137 (1996).

36) Nagai N, Ogata H. Quantitative relationship between pharmacokinetics of unchanged cisplatin and nephrotoxicity in rats: importance of area under the concentration-time curve $(A U C)$ as the major toxicodynamic determinant in vivo. Cancer Chemother. Pharmacol., 40, 11-18 (1997).

37) Arrieta O, Michel Ortega RM, Villanueva-Rodriguez G, SernaThome MG, Flores-Estrada D, Diaz-Romero C, Rodríguez CM, Martínez L, Sánchez-Lara K. Association of nutritional status and serum albumin levels with development of toxicity in patients with advanced non-small cell lung cancer treated with paclitaxel-cisplatin chemotherapy: a prospective study. BMC Cancer, 10, 50 (2010).

38) Willox JC, McAllister EJ, Sangster G, Kaye SB. Effects of magnesium supplementation in testicular cancer patients receiving cisplatin: a randomised trial. Br. J. Cancer, 54, 19-23 (1986).

39) Kidera Y, Kawakami H, Sakiyama T, Okamoto K, Tanaka K, Takeda M, Kaneda H, Nishina S, Tsurutani J, Fujiwara K, Nomura M, Yamazoe Y, Chiba Y, Nishida S, Tamura T, Nakagawa K. Risk factors for cisplatin-induced nephrotoxicity and potential of magnesium supplementation for renal protection. PLOS ONE, 9, e101902 (2014).

40) Murray MD, Brater DC. Renal toxicity of the nonsteroidal antiinflammatory drugs. Annu. Rev. Pharmacol. Toxicol., 33, 435-465 (1993).

41) Sendur MA, Aksoy S, Yaman S, Arik Z, Tugba Kos F, Akinci MB, Civelek B, Yildirim Ozdemir N, Uncu D, Zengin N. Administration of contrast media just before cisplatin-based chemotherapy increases cisplatin-induced nephrotoxicity. J. BUON, 18, 274-280 (2013).

42) Naughton CA. Drug-induced nephrotoxicity. Am. Fam. Physician, 78, 743-750 (2008). 